\title{
Association of arteriosclerosis and/or atherosclerosis with hypertensive target organ damage in the community-dwelling elderly Chinese: the Northern Shanghai Study
}

\author{
This article was published in the following Dove Press journal: \\ Clinical Interventions in Aging \\ 2 June 2017 \\ Number of times this article has been viewed
}

\section{Ximin Fan \\ Mengyun Zhu \\ Chen Chi \\ Shikai Yu \\ jing Xiong \\ Yuyan Lu \\ Bin Bai \\ Yawei Xu \\ Yi Zhang}

Department of Cardiology, Shanghai Tenth People's Hospital, Tongji University School of Medicine, Shanghai, People's Republic of China

Correspondence: Yi Zhang; Yawei Xu Department of Cardiology, Shanghai Tenth People's Hospital, Tongji University School of Medicine, $30 \mathrm{I}$ Yanchang Middle Road, Shanghai 200072,

People's Republic of China

Tel +862166306920

Fax +86 21 66307239

Email yizshcn@gmail.com;

xuyaweicn@aliyun.com
Objective: Vascular abnormality includes two forms, arteriosclerosis (ARS) and atherosclerosis (ATS), which coexist in patients with cardiovascular (CV) diseases. However, whether their combination may lead to a worsening status in those patients remains unclear. We therefore aimed to investigate the association of ARS and/or ATS with hypertensive target organ damage (TOD). Methods: From June 2014 to August 2015, a total of 1,599 community-dwelling elderly subjects (aged $>65$ years) from northern Shanghai were recruited. Vascular measurements, such as carotid-femoral pulse wave velocity (cf-PWV), ankle-brachial index (ABI) and carotid plaque, were conducted on each participant, and ARS was defined as cf-PWV $>12 \mathrm{~m} / \mathrm{s}$, while ATS was defined as participants who have carotid plaque or $\mathrm{ABI}<0.9$. Within the framework of comprehensive CV examinations, CV risk factors were assessed, and asymptomatic TOD was evaluated by measuring participants' left ventricular mass index (LVMI), peak transmitral pulsed Doppler velocity/early diastolic tissue Doppler velocity (E/Ea), urinary albumin-creatinine rate (UACR) and estimated glomerular filtration rate (eGFR).

Results: Although LVMI, E/Ea and eGFR were significantly different among subjects with or without ARS and/or ATS $(P<0.02)$, in full adjustment model, only E/Ea showed the independent and significant difference $(P=0.023)$. Moreover, E/Ea was significantly different between participants with ARS or ATS and those without ARS or ATS ( $P=0.045)$, while there was no significant difference between participants with ARS and ATS and those without ARS or ATS $(P=0.28)$. Similar results were obtained in the multivariate logistic regression of left ventricular diastolic dysfunction (LVDD). With similar adjustment, LVDD was significantly associated with $\operatorname{ATS}(P=0.01)$ but not with $\operatorname{ARS}(P=0.99)$.

Conclusion: In the community-dwelling elderly Chinese, among hypertensive TOD, LVDD was significantly associated with ATS but not with ARS. The proportion of patients with LVDD was not significantly different despite the presence of both ATS and ARS, when compared to patients with ATS alone.

Keywords: arteriosclerosis, atherosclerosis, target organ damage, pulse wave velocity, carotid plaque

\section{Introduction}

Many cardiovascular (CV) events and deaths are tightly associated with vascular structural and functional changes. From a pathophysiological point of view, vascular abnormality includes two forms; one is arteriosclerosis (ARS) and the other is atherosclerosis (ATS). Both are critical, fatal or event-related. ${ }^{1}$ 
Carotid-femoral pulse wave velocity (cf-PWV) is recognized as a gold standard for the assessment of patients' arterial stiffness, while carotid plaque detection by ultrasound and measurement of ankle-brachial index (ABI) by the four-limb blood pressure (BP) monitoring are the common methods to detect patients' ATS in routine clinical practice. ${ }^{2}$ Several reviews and articles have reported that patients may simultaneously suffer from ARS and ATS, and these two vascular disorders were tightly linked. ${ }^{3-6}$ For instance, a large population study with 3,000 elderly subjects aged 60-101 years reported that arterial stiffness was intensively associated with ATS ( $P$ for trend $<0.01$ ). ${ }^{7}$ Moreover, Huynh et $\mathrm{al}^{8}$ proposed that arterial stiffening may affect endothelial cells in the vessel wall with aging and promote atherogenesis. In addition, Demer suggested a relationship between functional stiffening and ATS progression. ${ }^{9}$ Therefore, it seems that ARS and ATS may commonly coexist in patients with cardiovascular diseases (CVD). Several previous studies have investigated the associations of ARS or ATS with some target organ damage (TOD). ${ }^{10-13}$ However, whether the combination of ARS and ATS would lead to a worsening prognosis or severe TOD remains unclear. Herein, we aimed to investigate the association of ARS and/or ATS with hypertensive TOD, within a framework of CV risk assessment in a communitydwelling elderly cohort.

\section{Methods}

\section{Study population}

The Northern Shanghai Study (NSS) is a communitydwelling prospective study. From June 2014 to August 2015, 1,721 residents were invited, of whom 1,599 (92.9\%) participants were enrolled. All residents were included if they were, 1) aged 65 years or more; 2) local residents from urban communities in the north of Shanghai, and 3) available for long-term follow-up. Exclusion criteria were as follows: 1) severe cardiac disease (New York Heart Association IV) or end-stage renal disease (chronic kidney disease $>4$ ); 2) malignant tumor with life expectancy $<5$ years, and 3) stroke history within 3 months. The NSS was conducted and financially supported by the Shanghai municipal government (Grant ID, 2013ZYJB0902) and had the approval of Shanghai Tenth People's Hospital Institutional Review Board. Written informed consent was obtained from all participants.

\section{Measurement of social, clinical and biological parameters}

Every participant was required to answer a questionnaire containing information such as gender, age, weight and height, smoking habits, family history of premature CVD, history of diabetes mellitus/hypertension/cardio-cerebrovascular diseases/renal diseases and usage of medications.

Venous blood samples and urine samples were obtained from fasting participants in the morning. Biological markers, including plasma low-density lipoprotein (LDL) cholesterol, high-density lipoprotein (HDL) cholesterol, plasma creatinine (PCr) and urinary microalbumin and creatinine, were assayed by standard methods in the Department of Laboratory Medicine of Shanghai Tenth People's Hospital. Fasting plasma glucose was measured by the glucose oxidase method. Estimated glomerular filtration rate (eGFR) was calculated by the modified MDRD (modification of diet in renal disease) formula for Chinese as follows: eGFR $\left(\mathrm{mL} / \mathrm{min} / 1.73 \mathrm{~m}^{2}\right)$ $=175 \times \mathrm{PCr}^{-1.234} \times$ age $^{-0.179}($ women $\times 0.79) .{ }^{14}$ Urinary microalbumin divided by urinary creatinine was defined as urinary albumin-creatinine ratio (UACR).

\section{Measurement of $\mathrm{BP}, \mathrm{ABI}$ and $\mathrm{PWV}$}

$\mathrm{BP}$ of each participant was measured three times in the morning using an electronic device, by specialized physicians, with at least 10 minutes' rest each time in a sitting position. The recorded BP value was calculated by the average of the three $\mathrm{BP}$ readings.

Bilateral brachial and ankle BPs were simultaneously and automatically measured to calculate $\mathrm{ABI}$ (the ratio of brachial systolic BP and ankle systolic BP) using the VP1000 system (Omron, Tokyo, Japan).

Cf-PWV was evaluated by one physician (who did not perform the ultrasound examination) using applanation tonometry (SphygmoCor; AtCor Medical, Sydney, Australia), according to the European Expert Consensus on Arterial Stiffness. ${ }^{15}$ Two pressure waves were recorded transcutaneously in the right carotid and right femoral arteries. PWV was identified as the foot-to-foot velocity. Pulse transit time for each artery was automatically calculated via electrocardiogram (ECG) data. The traveling distance covered by the pulse wave between the two recording sites was measured over the body surface. cf-PWV was calculated by traveling distance divided by traveling time, and an operator index $>80 \%$ indicated a high-quality measurement.

\section{Ultrasonography}

Ultrasound examinations were performed by a single experienced sonographer using the MyLab 30 Gold CV system (ESAOTE SpA, Genoa, Italy), with a $7.5 \mathrm{MHz}$ probe. The presence or absence of plaque in the right carotid artery was assessed by evaluating the ultrasonographic images. 
No attempt was made to record the quantity and size of the lesions. "Plaque $=0$ " referred to the absence of plaque, while "plaque $=1$ " referred to the presence of plaque.

M-mode or two-dimensional echocardiography was used to assess the measurement of left ventricular end-diastolic diameter (LVEDd), interventricular septal diameter (IVSd), posterior wall thickness at end-diastole diameter (PWTd) and left ventricular end-systolic diameter (LVESd) from the parasternal view, ${ }^{16,17}$ and then left ventricular mass (LVM) was calculated through those parameters using related formulas. ${ }^{18}$ Left ventricular mass index (LVMI) was assessed by LVM divided by body surface area (BSA). ${ }^{19}$ LVM $(\mathrm{g})=0.8 \times$ $\left\{1.04 \times\left[(\mathrm{LVEDd}+\mathrm{PWTd}+\mathrm{IVSd})^{3}-(\mathrm{LVEDd})^{3}\right]\right\}+0.6$ and LVMI $\left(\mathrm{g} / \mathrm{m}^{2}\right)=\mathrm{LVM} / \mathrm{BSA}$. Left ventricular ejection fraction (LVEF) was calculated through left ventricular end-diastolic volume (EDV) and end-systolic volume (ESV) estimates, using the following formula: $\mathrm{LVEF}=(\mathrm{EDV}-\mathrm{ESV}) /$ EDV ${ }^{20}$ Peak transmitral pulsed Doppler velocity/early diastolic tissue Doppler velocity (E/Ea) was performed by continual wave Doppler in the four-chamber view and was calculated for the evaluation of LV diastolic function. The abovementioned measurements were made according to the guidelines laid out by the American Society of Echocardiography (ASE). ${ }^{16}$

\section{Definition of ARS and ATS}

ARS was defined as cf-PWV $>12 \mathrm{~m} / \mathrm{s}$, while ABI $<0.9$ or the presence of carotid plaque represented ATS. ${ }^{2,21,22}$

\section{Definition of hypertensive TOD}

Asymptomatic hypertensive TOD investigated in this study included cardiac and renal TOD. Left ventricular hypertrophy (LVH) was defined as LVMI $\geq 115 \mathrm{~g} / \mathrm{m}^{2}$ (male) or LVMI $\geq 95 \mathrm{~g} / \mathrm{m}^{2}$ (female). Left ventricular diastolic dysfunction (LVDD) was assessed by E/Ea and other evidence of abnormal left ventricular relaxing and filling, such as enlarged left atrial volume and increased LVM. ${ }^{23}$ Specifically, diastolic dysfunction was defined as $\mathrm{E} / \mathrm{Ea} \geq 15$ or $15>\mathrm{E} / \mathrm{Ea}>8$ with the following (LVMI $>149 \mathrm{~g} / \mathrm{m}^{2}$ [male] or LVMI $\geq 122 \mathrm{~g} / \mathrm{m}^{2}$ [female]). ${ }^{23,24}$ Renal TOD was defined as microalbuminuria (MAU) (UACR > 30) and renal dysfunction $(\mathrm{RD})$ (creatinine clearance rate $<60 \mathrm{~mL} / \mathrm{min} / 1.73 \mathrm{~m}^{2}$ ).

\section{Statistical analysis}

Continuous variables were expressed as mean \pm standard deviation (SD), and categorical variables were presented as numbers with the percentage in parentheses. Participants were divided into three groups according to the participants' presence of ARS and/or ATS, such as normal (without ARS and
ATS), ARS/ATS (with either ARS or ATS) and ARS+ATS (with both ARS and ATS). The association of conventional $\mathrm{CV}$ risk factors with participants with or without ARS and/or ATS (normal, ARS/ATS, ARS+ATS) was assessed by general linear model for variance analysis, such as age, gender, body mass index (BMI), smoking status, systolic BP, fasting plasma glucose and LDL cholesterol. Three models were set with different adjustments: Model 1 (without adjustment), Model 2 (with adjustment for age and gender) and Model 3 (with adjustment for age, gender, BMI, smoking status, systolic $\mathrm{BP}$ and blood glucose). The comparisons of all parameters of asymptomatic TOD among the three groups were carried out using variance analysis, including LVMI, E/Ea, UACR and eGFR. Furthermore, multivariate logistic regressions were conducted to investigate the association of hypertensive TOD with ARS/ATS and ARS+ATS. Statistical analysis was performed using standard procedures from the Statistical Analysis System (SAS), version 9.3 (SAS Institute, Cary, NC, USA). $P<0.05$ was considered statistically significant.

\section{Results \\ Participants}

Characteristics of participants by gender are presented in Table 1, including conventional CV risk factors, asymptomatic hypertensive TOD and diseases and treatment. The 1,599 participants included 711 (44.5\%) men, 312 (19.5\%) diabetics and 843 (52.7\%) hypertensives, of whom 799 (93.9\%) were taking antihypertensive agents. Men, compared with women, had lower total cholesterol (4.92 \pm 0.99 vs $5.46 \pm 0.96 \mathrm{mmol} / \mathrm{L}, P<0.001)$, triglycerides $(1.54 \pm 0.85$ vs $1.66 \pm 1.00 \mathrm{mmol} / \mathrm{L}, P=0.008)$, HDL cholesterol $(1.28 \pm 0.33$ vs $1.46 \pm 0.36 \mathrm{mmol} / \mathrm{L}, P<0.001)$, LDL cholesterol (3.04 \pm 0.85 vs $3.33 \pm 0.83 \mathrm{mmol} / \mathrm{L}, P<0.001)$, pulse pressure $(54.4 \pm 14.0$ vs $56.2 \pm 16.1 \mathrm{mmHg}, P=0.02)$, eGFR $(88.3 \pm 20.0$ vs $\left.95.8 \pm 22.5 \mathrm{~mL} / \mathrm{min} / 1.73 \mathrm{~m}^{2}, P<0.001\right)$, E/Ea $(9.29 \pm 3.51$ vs $10.11 \pm 3.71, P<0.001)$ but they had significantly more smokers $(49.4 \%$ vs $1.7 \%, P<0.001)$ and greater incidence of carotid plaque ( $54.3 \%$ vs $48.9 \%, P=0.03)$.

\section{Association of ARS and/or ATS with CV risk factors}

Conventional $\mathrm{CV}$ risk factors, including age, gender, BMI, smoking status, systolic BP, fasting plasma glucose and LDL cholesterol, were put into a variance analysis model to investigate their association with ARS and ATS. As shown in Table 2, age, BMI, smoking status, systolic BP and fasting plasma glucose were significantly different between participants with or without ARS and/or ATS $(P \leq 0.02)$. 
Table I Characteristics of participants by gender

\begin{tabular}{|c|c|c|c|c|}
\hline Characteristics & Overall $(n=1,599)$ & Men (n=7II) & Women $(n=888)$ & $P$-value \\
\hline \multicolumn{5}{|l|}{$\mathrm{CV}$ risk factors } \\
\hline Age, years & $72.6 \pm 6.0$ & $72.7 \pm 5.9$ & $72.6 \pm 6.1$ & 0.96 \\
\hline Smoker, n (\%) & $366(22.9)$ & $351(49.4)$ & $15(1.7)$ & $<0.001$ \\
\hline $\mathrm{BMI}, \mathrm{kg} / \mathrm{m}^{2}$ & $23.9 \pm 3.5$ & $23.9 \pm 3.3$ & $23.9 \pm 3.6$ & 0.92 \\
\hline Fasting plasma glucose, $\mathrm{mmol} / \mathrm{L}$ & $5.69 \pm 1.70$ & $5.72 \pm 1.67$ & $5.67 \pm 1.73$ & 0.55 \\
\hline Total cholesterol, $\mathrm{mmol} / \mathrm{L}$ & $5.22 \pm 1.01$ & $4.92 \pm 0.99$ & $5.46 \pm 0.96$ & $<0.001$ \\
\hline Triglycerides, $\mathrm{mmol} / \mathrm{L}$ & $\mathrm{I} .6 \mathrm{I} \pm 0.93$ & $1.54 \pm 0.85$ & $1.66 \pm 1.00$ & 0.008 \\
\hline $\mathrm{HDL}$ cholesterol, mmol/L & $1.38 \pm 0.36$ & $1.28 \pm 0.33$ & $1.46 \pm 0.36$ & $<0.001$ \\
\hline LDL cholesterol, mmol/L & $3.20 \pm 0.85$ & $3.04 \pm 0.85$ & $3.33 \pm 0.83$ & $<0.001$ \\
\hline Systolic BP, mmHg & $134.3 \pm 17.7$ & $134.3 \pm 16.8$ & $134.3 \pm 18.4$ & 0.95 \\
\hline Diastolic BP, mmHg & $78.9 \pm 9.1$ & $80.0 \pm 9.2$ & $78.1 \pm 9.0$ & $<0.001$ \\
\hline \multicolumn{5}{|l|}{ Asymptomatic hypertensive TOD } \\
\hline Pulse pressure, $\mathrm{mmHg}$ & $55.4 \pm 15.2$ & $54.4 \pm 14.0$ & $56.2 \pm 16.1$ & 0.02 \\
\hline LVMI, $g / \mathrm{m}^{2}$ & $90.0 \pm 28.6$ & $90.5 \pm 28.8$ & $89.6 \pm 28.4$ & 0.54 \\
\hline Plaque in right carotid artery, $\mathrm{n}(\%)$ & $817(51.3)$ & $384(54.3)$ & $433(48.9)$ & 0.03 \\
\hline cf-PWV, m/s & $9.42 \pm 2.31$ & $9.36 \pm 2.42$ & $9.48 \pm 2.21$ & 0.32 \\
\hline eGFR, $\mathrm{mL} / \mathrm{min} / \mathrm{I} .73 \mathrm{~m}^{2}$ & $92.4 \pm 21.7$ & $88.3 \pm 20.0$ & $95.8 \pm 22.5$ & $<0.001$ \\
\hline UACR, mg/g & $54.9 \pm 181.6$ & $51.1 \pm 108.3$ & $58.0 \pm 224.4$ & 0.45 \\
\hline $\mathrm{E} / \mathrm{Ea}$ & $9.75 \pm 3.64$ & $9.29 \pm 3.51$ & $|0.11 \pm 3.7|$ & $<0.001$ \\
\hline Left $A B I, g / m^{2}$ & $1.03 \pm 0.13$ & $1.04 \pm 0.13$ & $1.03 \pm 0.13$ & 0.60 \\
\hline LVEF & $0.58 \pm 0.19$ & $0.59 \pm 0.10$ & $0.58 \pm 0.23$ & 0.23 \\
\hline \multicolumn{5}{|l|}{ Diseases and treatment } \\
\hline Hypertension, n (\%) & $843(52.7)$ & $385(54.2)$ & $458(5 \mathrm{I} .6)$ & 0.31 \\
\hline Treated hypertension, $\mathrm{n}(\%)$ & $799(93.9)$ & $363(93.1)$ & $436(94.6)$ & 0.36 \\
\hline CVD, n (\%) & $549(34.3)$ & $231(32.5)$ & $318(35.8)$ & 0.16 \\
\hline Anti-platelet treatment, n (\%) & $424(26.6)$ & $204(28.8)$ & $220(24.8)$ & 0.07 \\
\hline Statin treatment, n (\%) & $259(16.2)$ & $102(14.4)$ & $157(17.7)$ & 0.08 \\
\hline Stroke or TIA, n (\%) & $318(19.9)$ & $130(18.3)$ & I88 (2।.2) & 0.15 \\
\hline Renal disease, $\mathrm{n}(\%)$ & $132(8.3)$ & $55(7.8)$ & $77(8.7)$ & 0.52 \\
\hline Diabetes, n (\%) & $312(19.5)$ & $137(19.3)$ & $175(19.7)$ & 0.83 \\
\hline Antidiabetic treatment, n (\%) & $262(37.8)$ & 115 (37.7) & 147 (37.8) & 0.98 \\
\hline Insulin treatment, n (\%) & $5 \mathrm{I}(7.4)$ & $22(7.2)$ & $29(7.5)$ & 0.90 \\
\hline
\end{tabular}

Notes: Data presented as mean \pm standard deviation unless stated otherwise. Student's $t$-test and chi-square test were conducted to compare the differences between men and women for quantitative and qualitative variables, respectively. eGFR was calculated using the modified MDRD formula for Chinese. E/Ea, peak transmitral pulsed Doppler velocity/early diastolic tissue Doppler velocity.

Abbreviations: ABI, ankle-brachial index; BMI, body mass index; BP, blood pressure; cf-PWV, carotid-femoral pulse wave velocity; CV, cardiovascular; CVD, cardiovascular disease; eGFR, estimated glomerular filtration rate; HDL, high-density lipoprotein; LDL, low-density lipoprotein; LVEF, left ventricular ejection fraction; LVMI, left ventricular mass index; MDRD, modification of diet in renal disease; SD, standard deviation; TIA, transient ischemic attack; TOD, target organ damage; UACR, urinary albumin-creatinine ratio.

Association of asymptomatic hypertensive TOD with ARS and/or ATS

In the variance analysis of ARS and ATS with hypertensive TOD, all parameters of asymptomatic TOD $(P<0.02)$ were significantly different among the three groups, except LVEF $(P=0.11$; Table 3, Model 1) and UACR $(P=0.16$; Table 3, Model 1). Of note, in Model 3 with full adjustment, only E/Ea significantly differed among the three groups $(P=0.02$;

Table 2 Association of CV risk factors with ARS and/or ATS

\begin{tabular}{|c|c|c|c|c|}
\hline CV risk factors & Normal $(n=622)$ & ARS/ATS $(n=792)$ & ARS + ATS $(n=128)$ & $P$-value \\
\hline Age, years & $71.2 \pm 5.11$ & $73.0 \pm 6.17$ & $76.6 \pm 6.35$ & $<0.001$ \\
\hline Gender (male), n (\%) & $256(4 \mid .2)$ & $368(46.5)$ & $53(4 I .4)$ & 0.11 \\
\hline BMI & $23.6 \pm 3.52$ & $24.0 \pm 3.44$ & $24.4 \pm 3.21$ & 0.02 \\
\hline Smoker, n (\%) & $114(18.3)$ & $210(26.5)$ & $28(21.9)$ & 0.001 \\
\hline Systolic BP, mmHg & $|3| .4 \pm 16.7$ & $135.2 \pm 17.7$ & $145.2 \pm 18.6$ & $<0.001$ \\
\hline Fasting plasma glucose, $\mathrm{mmol} / \mathrm{L}$ & $5.56 \pm 1.6 \mathrm{I}$ & $5.69 \pm 1.74$ & $6.39 \pm 1.88$ & $<0.001$ \\
\hline LDL cholesterol, $\mathrm{mmol} / \mathrm{L}$ & $3.16 \pm 0.78$ & $3.22 \pm 0.89$ & $3.28 \pm 0.91$ & 0.24 \\
\hline
\end{tabular}

Notes: Data presented as mean \pm standard deviation unless stated otherwise. Variance analysis was done to investigate the association of CV risk factors with ARS and/or ATS; ARS/ATS, with either ARS or ATS; ARS+ATS, with both ARS and ATS.

Abbreviations: ARS, arteriosclerosis; ATS, atherosclerosis; BMI, body mass index; BP, blood pressure; CV, cardiovascular; LDL, low-density lipoprotein. 
Table 3 Association of ARS and/or ATS with parameters of asymptomatic hypertensive TOD analyzed by variance analyses with different adjustments

\begin{tabular}{lllllll}
\hline $\begin{array}{l}\text { Asymptomatic } \\
\text { hypertensive TOD }\end{array}$ & $\begin{array}{l}\text { Normal } \\
(\mathbf{n}=\mathbf{6 2 2})\end{array}$ & $\begin{array}{l}\text { ARS/ATS } \\
(\mathbf{n}=\mathbf{7 9 2})\end{array}$ & $\begin{array}{l}\text { ARS+ATS } \\
(\mathbf{n}=\mathbf{1 2 8})\end{array}$ & $\begin{array}{l}\text { Model I, } \\
\boldsymbol{P} \text {-value }\end{array}$ & $\begin{array}{l}\text { Model 2, } \\
\boldsymbol{P} \text {-value' }\end{array}$ & $\begin{array}{l}\text { Model 3, } \\
\boldsymbol{P} \text {-value" }\end{array}$ \\
\hline LVMI, g/m $\mathrm{m}^{2}$ & $86.2 \pm 26.6$ & $91.4 \pm 29.7$ & $96.8 \pm 25.0$ & $<0.00 \mathrm{I}$ & 0.004 & 0.07 \\
E/Ea & $9.4 \pm 3.31$ & $10.0 \pm 3.91$ & $9.8 \pm 3.34$ & 0.02 & 0.02 & 0.02 \\
LVEF & $0.57 \pm 0.27$ & $0.59 \pm 0.10$ & $0.57 \pm 0.11$ & $0.1 \mathrm{I}$ & 0.12 & 0.10 \\
UACR, $\mathrm{mg} / \mathrm{g}$ & $45.8 \pm 85.0$ & $56.2 \pm 235.3$ & $79.2 \pm 147.9$ & 0.16 & 0.34 & 0.76 \\
eGFR, $\mathrm{mL} / \mathrm{min} / \mathrm{l} .73 \mathrm{~m}^{2}$ & $94.8 \pm 20.1$ & $92.3 \pm 22.5$ & $85.9 \pm 22.8$ & $<0.001$ & 0.34 & 0.08 \\
\hline
\end{tabular}

Notes: Data presented as mean \pm standard deviation unless stated otherwise. Variance analyses with different adjustments were conducted to investigate the association of ARS and/or ATS with parameters of asymptomatic hypertensive TOD. E/Ea, peak transmitral pulsed Doppler velocity/early diastolic tissue Doppler velocity; Model I: without adjustment; Model 2: with adjustment for age and gender; Model 3: with adjustment for age, gender, BMl, smoking status, systolic BP and blood glucose. ARS/ATS, with either ARS or ATS; ARS+ATS, with both ARS and ATS.

Abbreviations: ARS, arteriosclerosis; ATS, atherosclerosis; eGFR, estimated glomerular filtration rate; LVEF, left ventricular ejection fraction; LVMI, left ventricular mass index; TOD, target organ damage; UACR, urinary albumin-creatinine ratio.

Table 3, Model 3). Moreover, E/Ea was significantly different between ARS/ATS and normal $(P=0.045)$, while there was no significant difference between ARS+ATS and normal $(P=0.28)$. As LVEF was not significantly different among these groups ( $P=0.10$; Table 3, Model 3 ), we did not adjust for LVEF in the subsequent multivariate logistic regressions.

As shown in Table 4, with similar adjustment strategy analyzed by the multiple logistic regression model, most asymptomatic TOD was significantly associated with ARS/ATS and ARS+ATS, but only ARS/ATS was significantly associated with LVDD in the full adjustment model, with an odds ratio $(\mathrm{OR})=1.50(95 \%$ confidence interval $[\mathrm{CI}]$ 1.07-2.09; Figure 1).

Finally, when ARS and ATS were separately put in the logistic regression model, in the full adjustment model, LVDD was significantly associated with ATS (OR 1.55; $95 \%$ CI 1.11-2.17, $P=0.01$ ) but not with ARS (OR 0.99; 95\% CI $0.45-2.18, P=0.99$ ) (Table 5).

\section{Discussion}

There are two major findings in this study. First, age, BMI, smoking status, systolic BP and fasting plasma glucose were significantly different between participants with or without ARS and/or ATS. Second, among hypertensive TOD, LVDD as measured by E/Ea was significantly associated with ATS but not with ARS, and the proportion of patients with LVDD was not significantly different despite the presence of both ATS and ARS, when compared to patients with ATS alone in the community-dwelling elderly Chinese.

ARS and ATS are pathologically different and possess different clinical manifestations. ${ }^{25,26}$ ARS is a degenerative process of the media of elastic arteries and the calcification in arterial wall under the effect of aging and risk factors. ${ }^{27}$ The mechanism of ARS is the chronic vascular inflammation derived from mitochondrial oxidative stress in smooth muscle cells. ${ }^{28}$ It would only cause stiffness and thickness of the arterial wall but without luminal stenosis and syndromes

Table 4 Association of ARS and/or ATS with parameters of asymptomatic hypertensive TOD analyzed by multivariate logistic regressions with different adjustments

\begin{tabular}{|c|c|c|c|}
\hline TOD & Model I OR $(95 \% \mathrm{CI})$ & Model 2 OR $(95 \% \mathrm{Cl})$ & Model 3 OR $(95 \% \mathrm{Cl})$ \\
\hline \multicolumn{4}{|l|}{ LVH } \\
\hline ARS/ATS vs normal & $1.21(0.95-1.53)$ & $1.21(0.95-1.55)$ & $1.18(0.92-1.52)$ \\
\hline ARS+ATS vs normal & $1.93(1.30-2.88)$ & $1.71(1.12-2.61)$ & $1.49(0.95-2.33)$ \\
\hline \multicolumn{4}{|l|}{ LVDD } \\
\hline ARS/ATS vs normal & $1.63(1.19-2.24)$ & $1.63(1.18-2.25)$ & $1.50(1.07-2.09)$ \\
\hline ARS+ATS vs normal & $1.76(1.03-3.00)$ & $1.47(0.85-2.57)$ & $1.06(0.59-1.90)$ \\
\hline \multicolumn{4}{|l|}{ MAU } \\
\hline ARS/ATS vs normal & $1.08(0.87-1.34)$ & $1.03(0.83-1.28)$ & $0.93(0.74-1.17)$ \\
\hline ARS+ATS vs normal & $1.99(1.35-2.94)$ & $1.63(1.09-2.44)$ & I. $18(0.77-1.81)$ \\
\hline \multicolumn{4}{|l|}{ RD } \\
\hline ARS/ATS vs normal & $2.03(1.26-3.29)$ & $1.63(0.99-2.68)$ & $1.63(0.99-2.70)$ \\
\hline ARS+ATS vs normal & $2.97(1.48-5.97)$ & $1.55(0.74-3.23)$ & $1.60(0.75-3.40)$ \\
\hline
\end{tabular}

Notes: Multivariate logistic regressions with different adjustments were conducted to investigate the association of ARS and/or ATS with asymptomatic hypertensive TOD. Model I: without adjustment; Model 2: with adjustment for age and gender; Model 3: with adjustment for age, gender, BMI, smoking status, systolic BP and blood glucose. ARS/ATS, with either ARS or ATS; ARS+ATS, with both ARS and ATS.

Abbreviations: ARS, arteriosclerosis; ATS, atherosclerosis; CI, confidence interval; LVH, left ventricular hypertrophy; LVDD, left ventricular diastolic dysfunction; MAU, microscale albuminuria; $\mathrm{OR}$, odds ratio; RD, renal dysfunction; TOD, target organ damage. 


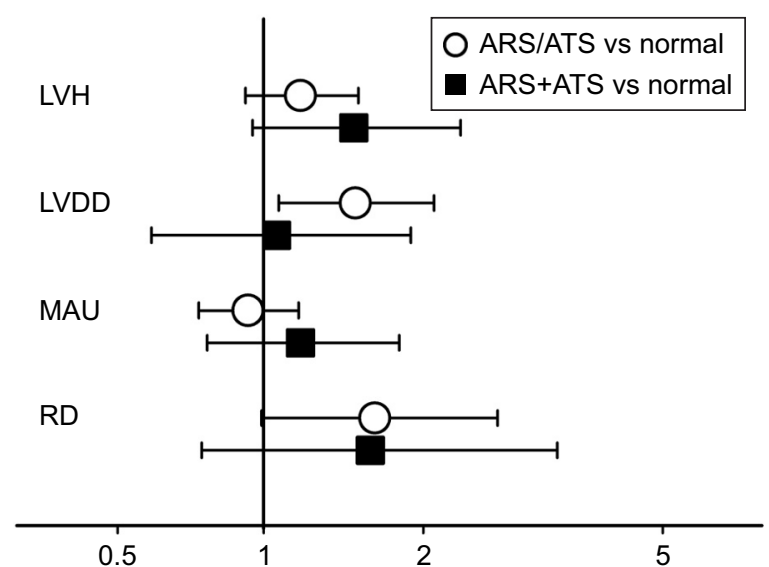

Figure I Association of ARS and/or ATS with asymptomatic hypertensive TOD in the full adjustment model.

Notes: Multivariate logistic regressions were conducted to investigate the association of ARS and/or ATS with asymptomatic hypertensive TOD with adjustment for age, gender, BMI, smoking status, systolic BP and blood glucose. Symbols $(\mathrm{O} / \mathbf{\square})$ and solid lines indicate OR and $95 \% \mathrm{Cl}$, respectively. ARS/ATS, with either ARS or ATS; ARS+ATS, with both ARS and ATS.

Abbreviations: ARS, arteriosclerosis; ATS, atherosclerosis; BMI, body mass index; $\mathrm{Cl}$, confidence interval; LVDD, left ventricular diastolic dysfunction; LVH, left ventricular hypertrophy; MAU, microalbuminuria; $O R$, odds ratio; RD, renal dysfunction; TOD, target organ damage.

of tissue/organ ischemia. ${ }^{4,29}$ However, ATS is the underlying cause of most ischemic diseases, such as myocardial infarction and ischemic stroke. Pathologically, ATS is a chronic inflammatory response to the accumulation of lipid in the artery wall and causes intimal plaques and stenosis in arteries. Disruption of atherosclerotic plaques will trigger the formation of a thrombus and subsequently cause acute ischemia of the end organ. Although its prevalence is also age-related, the major risk factor that promotes the development of ATS is high level of cholesterol. ${ }^{30,31}$ As ARS and ATS are the two most common types of modifications in vascular structure and they are prevalent in the elderly, it is meaningful to investigate their respective and combined association with TOD. In this study, we found that, among cardiac and renal TOD, only LVDD was significantly associated with ATS but not with ARS. The association of LVDD with different indicators of arterial stiffness and ATS has been previously reported. Some previous studies indicated that arterial stiffness, assessed by the cardio-ankle vascular index (CAVI), was significantly correlated with LVDD. ${ }^{32-34}$ Some other reports discussed a strong association between LVDD and arterial stiffness assessed by PWV. ${ }^{35,36}$ Albu et $\mathrm{al}^{37}$ conducted an investigation into 96 postmenopausal women and proposed that aortic PWV was an independent predictor of LVDD (OR 2.15, 95\% CI 1.39-3.31, $P=0.0006$ ), while carotid plaque score did not show correlation with LVDD after adjustment. However, some investigators reported that the presence of carotid ATS was significantly related to LVDD, ${ }^{38-40}$ which was in accordance with our finding. Other studies concerning arterial stiffness were not consistent with our results. Several possible reasons may probably contribute to the discrepancies. First, arterial stiffness in those studies was assessed mainly by the CAVI but not by PWV. Although the CAVI, as a new index of arterial stiffness, was suggested to be superior to PWV due to its BP independence, different indicators in arterial stiffness may lead to miscellaneous results. Second, those studies were mainly conducted on patients with high $\mathrm{CV}$ risk or with coronary artery disease (CAD) or heart failure (HF) or other established cardiac diseases, while we focused on the community-dwelling elderly population. The last point may be the most important. Since participants in those studies may suffer both ATS and ARS, the observed significant association between arterial stiffness of those participants and LVDD would be due to ATS, and the association was therefore overestimated. However, in our analysis, ARS and ATS were separately investigated.

Nevertheless, there are limited data concerning the mutual association of ARS and ATS with LVDD. In our study, we found that their combination did not actually worsen patients' LV diastolic function. This finding seems to conflict with the finding that LVDD was significantly associated with ATS. Of note, since our results were double checked by the variance analysis and multivariate logistic regression models and with sufficient adjustments, we believe that our findings are reliable. The possible explanation may lie in the fact that arterial stiffening is very prevalent in the elderly. Therefore, PWV, as influenced by biological aging, become

Table 5 Association of ARS and ATS with LVDD analyzed by multivariate logistic regressions with different adjustments

\begin{tabular}{llll}
\hline TOD & Model I OR $(\mathbf{9 5} \% \mathbf{C I})$ & Model 2 OR $(\mathbf{9 5} \% \mathbf{~ C l})$ & Model 3 OR (95\% CI) \\
\hline LVDD & & & \\
ARS vs normal & $1.53(0.72-3.24)$ & $1.32(0.6 I-2.84)$ & $0.99(0.45-2.18)$ \\
ATS vs normal & $1.64(1.19-2.26)$ & $1.65(1.19-2.29)$ & $1.55(1.11-2.17)$ \\
ARS+ATS vs normal & $1.76(1.03-3.00)$ & $1.47(0.84-2.56)$ & $1.04(0.58-1.88)$ \\
\hline
\end{tabular}

Notes: Multivariate logistic regressions with different adjustments were conducted to investigate the respective association of ARS and ATS with LVDD. Model I: without adjustment; Model 2: with adjustment for age and gender; Model 3: with adjustment for age, gender, BMI, smoking status, systolic BP and blood glucose. ARS+ATS, with both ARS and ATS.

Abbreviations: ARS, arteriosclerosis; ATS, atherosclerosis; BMI, body mass index; BP, blood pressure; CI, confidence interval; OR, odds ratio; LVDD, left ventricular diastolic dysfunction; TOD, target organ damage. 
less significant in predicting events and deaths in the geriatric population. ${ }^{41-43}$ Further investigations focusing on the younger population or middle-age population are warranted to confirm the present finding.

Since hypertensive TOD is of great importance and is recognized as the intermediate outcome connecting $\mathrm{CV}$ risk factors and CV events and mortality, it is meaningful to investigate some potential markers to screen TOD. LVDD is considered as one of the major causes of HF with preserved ejection fraction. Our finding indicated that, in accordance with other studies, LVDD was tightly associated with ATS. Therefore, the presence of ATS, even in the elderly, may indicate the high risk for the development of LVDD and early and intensified intervention may be needed in preventing future heart failure with preserved ejection fraction.

\section{Limitations}

Findings of this study need to be interpreted within the context of its limitations. First, we could not fully adjust for the potential influence from the various medications on cf-PWVs, ABI and carotid plaque, etc., such as antihypertensive, antidiabetic and anti-hyperlipidemic drugs. Second, as a cross-sectional analysis, this study cannot tell the causeeffect relationship between ATS and LVDD.

\section{Conclusion}

In the community-dwelling elderly Chinese, among hypertensive TOD, LVDD was significantly associated with ATS but not with ARS. The proportion of patients with LVDD was not significantly different despite the presence of both ATS and ARS, when compared to patients with ATS alone.

\section{Acknowledgments}

We thank all the investigators, staff and patients involved in this study for their assistance and cooperation. Jiadela Teliewubai and Yiwu Zhou provided purely technical help, while Jun Zhang and Hongwei Ji provided writing assistance. This study was authorized and financially supported by the Shanghai municipal government (Grant IDs, 2013ZYJB0902; 15GWZK1002). Dr Yi Zhang was supported by the National Nature Science Foundation of China (Grant IDs, 81300239; 81670377).

\section{Disclosure}

The authors report no conflicts of interest in this work.

\section{References}

1. Benetos A, Safar M, Rudnichi A, et al. Pulse pressure: a predictor of long-term cardiovascular mortality in a French male population. Hypertension. 1997;30(6):1410-1415.
2. Zureik M, Bureau JM, Temmar M, et al. Echogenic carotid plaques are associated with aortic arterial stiffness in subjects with subclinical carotid atherosclerosis. Hypertension. 2003;41(3):519-527.

3. Oberoi S, Schoepf UJ, Meyer M, et al. Progression of arterial stiffness and coronary atherosclerosis: longitudinal evaluation by cardiac CT. AJR Am J Roentgenol. 2013;200(4):798-804.

4. Palombo C, Kozakova M. Arterial stiffness, atherosclerosis and cardiovascular risk: pathophysiologic mechanisms and emerging clinical indications. Vascul Pharmacol. 2016;77:1-7.

5. Vakhovskaia TV, Balakhonova TV, Luk'ianov MM, Titov VN, Masenko VP, Boitsov SA. [Arterial stiffness and blood levels of glycation end-products in patients with arterial hypertension and carotid atherosclerosis]. Klin Med (Mosk). 2013;91(5):4-9.

6. Sudano I, Roas S, Noll G. Vascular abnormalities in essential hypertension. Curr Pharm Des. 2011;17(28):3039-3044.

7. van Popele NM, Grobbee DE, Bots ML, et al. Association between arterial stiffness and atherosclerosis: the Rotterdam Study. Stroke. 2001;32(2): 454-460.

8. Huynh J, Nishimura N, Rana K, et al. Age-related intimal stiffening enhances endothelial permeability and leukocyte transmigration. Sci Transl Med. 2011;3(112):112ra122.

9. Demer LL. Effect of calcification on in vivo mechanical response of rabbit arteries to balloon dilation. Circulation. 1991;83(6):2083-2093.

10. Briet M, Boutouyrie P, Laurent S, London GM. Arterial stiffness and pulse pressure in CKD and ESRD. Kidney Int. 2012;82(4):388-400.

11. Kollias A, Stergiou GS, Dolan E, O’Brien E. Ambulatory arterial stiffness index: a systematic review and meta-analysis. Atherosclerosis. 2012; 224(2):291-301.

12. Triantafyllidi H, Tzortzis S, Lekakis J, et al. Association of target organ damage with three arterial stiffness indexes according to blood pressure dipping status in untreated hypertensive patients. Am J Hypertens. 2010;23(12):1265-1272.

13. Roman MJ, Pickering TG, Schwartz JE, Pini R, Devereux RB. Association of carotid atherosclerosis and left ventricular hypertrophy. J Am Coll Cardiol. 1995;25(1):83-90.

14. Ma YC, Zuo L, Chen JH, et al; Chinese eGFR Investigation Collaboration. Improved GFR estimation by combined creatinine and cystatin $\mathrm{C}$ measurements. Kidney Int. 2007;72(12):1535-1542.

15. Van Bortel LM, Laurent S, Boutouyrie P, et al; Artery Society; European Society of Hypertension Working Group on Vascular Structure and Function; European Network for Noninvasive Investigation of Large Arteries. Expert consensus document on the measurement of aortic stiffness in daily practice using carotid-femoral pulse wave velocity. J Hypertens. 2012;30(3):445-448.

16. Lang RM, Bierig M, Devereux RB, et al; American Society of Echocardiography's Nomenclature and Standards Committee; Task Force on Chamber Quantification; American College of Cardiology Echocardiography Committee; American Heart Association; European Association of Echocardiography, European Society of Cardiology. Recommendations for chamber quantification. Eur $J$ Echocardiogr. 2006;7(2):79-108

17. Zhang Y, Protogerou AD, Iaria P, Safar ME, Xu Y, Blacher J. Prognosis in the hospitalized very elderly: the PROTEGER study. Int J Cardiol. 2013;168(3):2714-2719.

18. Devereux RB, Alonso DR, Lutas EM, et al. Echocardiographic assessment of left ventricular hypertrophy: comparison to necropsy findings. Am J Cardiol. 1986;57(6):450-458.

19. Brilla CG. [What is the value of the left ventricular muscle mass index (LVMI) in echocardiographic diagnosis of left heart hypertrophy?] Internist (Berl). 1993;34(10):986.

20. Lang RM, Badano LP, Mor-Avi V, et al. Recommendations for cardiac chamber quantification by echocardiography in adults: an update from the American Society of Echocardiography and the European Association of Cardiovascular Imaging. J Am Soc Echocardiogr. 2015;28(1):1.e-39.e.

21. Asmar R, Benetos A, Topouchian J, et al. Assessment of arterial distensibility by automatic pulse wave velocity measurement. Validation and clinical application studies. Hypertension. 1995;26(3):485-490. 
22. Carter SA. Indirect systolic pressures and pulse waves in arterial occlusive diseases of the lower extremities. Circulation. 1968;37(4): 624-637.

23. Zhang Y, Kollias G, Argyris AA, et al. Association of left ventricular diastolic dysfunction with 24-h aortic ambulatory blood pressure: the SAFAR study. J Hum Hypertens. 2015;29(7):442-448.

24. Kane GC, Karon BL, Mahoney DW, et al. Progression of left ventricular diastolic dysfunction and risk of heart failure. JAMA. 2011;306(8): 856-863.

25. Wilkinson IB, McEniery CM, Cockcroft JR. Arteriosclerosis and atherosclerosis: guilty by association. Hypertension. 2009;54(6): 1213-1215.

26. Pickering G. Arteriosclerosis and atherosclerosis. The need for clear thinking. Am J Med. 1963;34:7-18.

27. Spronck B, Heusinkveld MH, Vanmolkot FH, et al. Pressure-dependence of arterial stiffness: potential clinical implications. J Hypertens. 2015;33(2):330-338.

28. Ungvari Z, Kaley G, de Cabo R, Sonntag WE, Csiszar A. Mechanisms of vascular aging: new perspectives. J Gerontol A Biol Sci Med Sci. 2010; 65(10):1028-1041.

29. Zhang J, Li Y, Wang Y, et al. Arterial stiffness and asymptomatic intracranial large arterial stenosis and calcification in hypertensive Chinese. Am J Hypertens. 2011;24(3):304-309.

30. Otsuka F, Kramer MC, Woudstra P, et al. Natural progression of atherosclerosis from pathologic intimal thickening to late fibroatheroma in human coronary arteries: a pathology study. Atherosclerosis. 2015; 241(2):772-782

31. Goldman L, Schafer AI. Goldman's Cecil Medicine. 24th ed. Philadelphia, PA: Elsevier; 2011:409-411. ISBN 978-1-4377-1604-7.

32. Namba T, Masaki N, Matsuo Y, et al. Arterial stiffness is significantly associated with left ventricular diastolic dysfunction in patients with cardiovascular disease. Int Heart J. 2016;57(6):729-735.

33. Masugata H, Senda S, Goda F, et al. Tissue Doppler echocardiography for predicting arterial stiffness assessed by cardio-ankle vascular index. Tohoku J Exp Med. 2009;217(2):139-146.

34. Sakane K, Miyoshi T, Doi M, et al. Association of new arterial stiffness parameter, the cardio-ankle vascular index, with left ventricular diastolic function. J Atheroscler Thromb. 2008;15(5):261-268.
35. Mizuguchi Y, Oishi Y, Tanaka H, et al. Arterial stiffness is associated with left ventricular diastolic function in patients with cardiovascular risk factors: early detection with the use of cardio-ankle vascular index and ultrasonic strain imaging. J Card Fail. 2007;13(9):744-751.

36. Eren M, Gorgulu S, Uslu N, Celik S, Dagdeviren B, Tezel T. Relation between aortic stiffness and left ventricular diastolic function in patients with hypertension, diabetes, or both. Heart. 2004;90(1):37-43.

37. Albu A, Fodor D, Bondor C, Poanta L. Arterial stiffness, carotid atherosclerosis and left ventricular diastolic dysfunction in postmenopausal women. Eur J Intern Med. 2013;24(3):250-254.

38. Harada M, Tabako S. Carotid atherosclerosis is associated with left ventricular diastolic function. J Echocardiogr. 2016;14(3):120-129.

39. Chahal NS, Lim TK, Jain P, Chambers JC, Kooner JS, Senior R. The distinct relationships of carotid plaque disease and carotid intimamedia thickness with left ventricular function. J Am Soc Echocardiogr. 2010;23(12):1303-1309.

40. Mizuguchi Y, Tanaka H, Oishi Y, et al. Predictive value of associations between carotid arterial sclerosis and left ventricular diastolic dysfunction in patients with cardiovascular risk factors. J Am Soc Echocardiogr. 2007;20(7):806-812.

41. Ben-Shlomo Y, Spears M, Boustred C, et al. Aortic pulse wave velocity improves cardiovascular event prediction: an individual participant meta-analysis of prospective observational data from 17,635 subjects. J Am Coll Cardiol. 2014;63(7):636-646

42. Miljkovic D, Perret-Guillaume C, Alla F, Salvi P, Erpelding ML, Benetos A. Correlation between peripheral blood pressure and pulse-wave velocity values in the institutionalized elderly persons 80 years of age and older: the PARTAGE study. Am J Hypertens. 2013;26(2):163-173.

43. Benetos A, Gautier S, Labat C, et al. Mortality and cardiovascular events are best predicted by low central/peripheral pulse pressure amplification but not by high blood pressure levels in elderly nursing home subjects: the PARTAGE (predictive values of blood pressure and arterial stiffness in institutionalized very aged population) study. J Am Coll Cardiol. 2012;60(16):1503-1511.
Clinical Interventions in Aging

\section{Publish your work in this journal}

Clinical Interventions in Aging is an international, peer-reviewed journal focusing on evidence-based reports on the value or lack thereof of treatments intended to prevent or delay the onset of maladaptive correlates of aging in human beings. This journal is indexed on PubMed Central, MedLine,

\section{Dovepress}

CAS, Scopus and the Elsevier Bibliographic databases. The manuscript management system is completely online and includes a very quick and fair peer-review system, which is all easy to use. Visit http://www.dovepress. com/testimonials.php to read real quotes from published authors. 